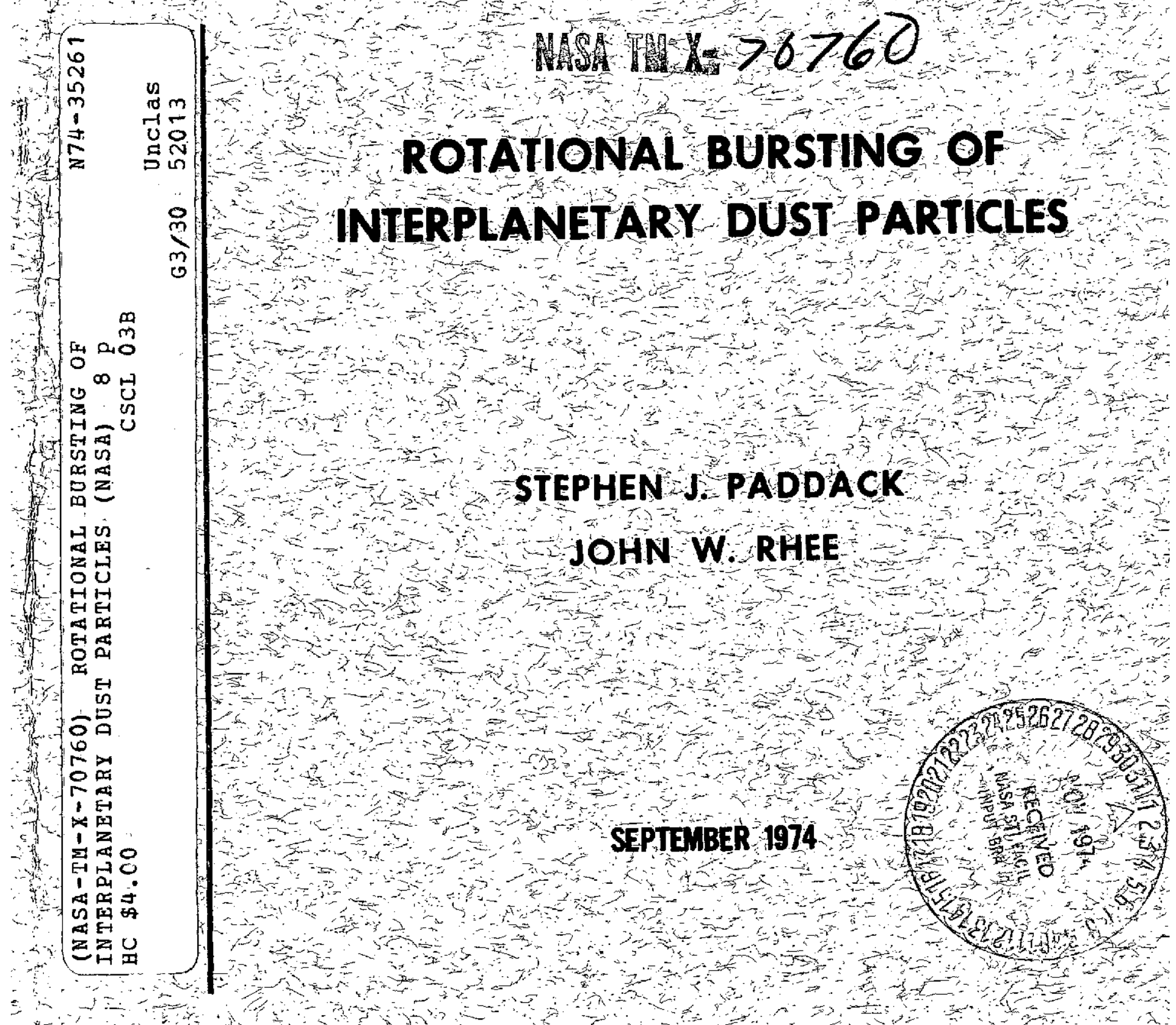


"This paper presents the views of the author(s), and does not necessarily reflect the views of the Goddard Space Flight Center, or NASA."'

For information concerning availability of this document contact:

Technical Information Division, Code 250

Goddard Space. Flight Center

Greenbelt, Maryland 20771

(Telephone 301-982-4488) 
$\mathrm{X}-405-74-300$

Preprint

\title{
ROTATIONAL BURSTING \\ OF INTERPLANETARY DUST PARTICLES
}

\author{
Stephen J. Paddack \\ and \\ John W. Rhee \\ $r$ \\ $a$
}

September 1974

To Be Submitted To: Geophysical Research Letters

GODDARD SPACE FLIGHT CENTER

Greenbelt, Maryland 


\section{ROTATIONAL BURSTING \\ OF INTERPLANETARY DUST PARTICLES}

Stephen J. Paddack

John W. Rhee

Abstract. Solar radiation pressure can cause rotational bursting and eventual elimination from the solar system of asymmetric dust particles by a windmill effect. The life span against this process for metallic particles with radii of $10^{-5}$ to $10^{-2} \mathrm{~cm}$ ranges from 10 to 10,000 years. The effects of magnetic spin damping have been considered in this estimate. This depletion mechanism works faster than the traditional Poynting-Robertson effect by approximately one order of magntidue for metallic particles and about two orders of magnitude for nonmetallic particles.

\section{Introduction}

Kresák (1968) has drawn attention to a puzzling deficiency in the numbers of small meteors. From the laws of celestial mechanics he notes that the breakup time of meteor showers is of the order of $10^{3}$ to $10^{5}$ years. After the breakup of the showers, the meteors will continue to exist as individuals in individual orbits (sporadic meteors) until they are destroyed. The fact that there are only about five times as many sporadic meteors as shower meteors means that the lifetime of meteors as solid objects must have a range of $5 \times 10^{3}$ to $5 \times 10^{5}$ years. But lifetime against the Poynting-Robertson effect is about $10^{7}$ years 
for a $1 \mathrm{~cm}$ particle. Hence some other more effective mechanism of destruction must exist. We here suggest that it is rotational bursting.

Radzievskii (1954) suggested that solar radiation pressure could cause rotational bursting of asteroids and meteorites having irregular albedos over their entire surfaces. As an example he calculated that a one centimeter granite cube at $0,4 \mathrm{~A}$. U. from the sun would reach bursting speed in about 1,000 years.

Paddack $(1969,1973)$ demonstrated that rotational bursting could be caused by the interaction of solar radiation pressure and irregular surface geometry; irrespective of variations in albedo.

On the basis of experimentation it was conservatively estimated that nonmagnetic meteorite and tektites in heliocentric orbit reach bursting speed in about 60,000 years. These analyses did not take into account magnetic spin damping effects for tektites because of their high electrical resistivity (Hoyte et al., 1965) and low magnetic permeability. However, since interplanetary dust particles are probably pieces of iron or stone, most of them are subject to magnetic spin damping.

\section{Analytical Procedure}

An irregularly shaped metallic body can also reach rotational bursting speed by this windmill effect. Applying the experimentally determined asymetry factor, $\ell$ which is $0.05 \%$ of the maximum dimension of the body (Paddack, 1969, 
1973) and using the radiation pressure, $P_{r}$ at one $A . U ., 4.5 \times 10^{-5}$ dynes $\mathrm{cm}^{-2}$, where $A$ is the total projected area, the effective torque, $N_{1}$, due to radiation pressure is given by

$$
\mathrm{N}_{1}=0.1 \mathrm{P}_{\mathrm{r}} \mathrm{Al}
$$

The fact that the angular speed of a conductor is reduced in an external magnetic field is a well known phenomenon. The magnitude of the retarding torque for a rotating sphere having angular velocity $\omega$, and radius $b$ is given by Smythe (1950).

$$
N_{2}=\frac{-2 \pi B^{2} b^{5} \omega}{3 \rho}
$$

where $\rho$ is the resistivity and $\mathrm{B}$ is the component of the interplanetary magnetic field intensity in the direction perpendicular to the spin axis. The skin depth effect as regards magntic spin damping has been taken into consideration but does not have an appreciable contribution for dust particles. Consequently, it is possible to express the rotational equation of motion for the interplanetary dust partcles including the damping effect due to interplanetary magnetic field.

$$
\mathrm{I} \frac{\mathrm{d} \omega}{\mathrm{dt}}=\mathrm{N}_{1}+\mathrm{N}_{2}
$$

where I is the moment of inertia. The solution of Equation 3 is

$$
\omega=\frac{\alpha}{\beta}\left(1-\mathrm{e}^{-\beta \mathrm{t}}\right)
$$

where $\alpha=\mathrm{N}_{1} / \mathrm{I}$ and $\beta=-\mathrm{N}_{2} / \mathrm{I} \omega$. Equation 4 shows that the angular motion is stabilized under the damping effect and its upper limit is $\alpha / \beta$. 
According to Timoshenko (1942), the stress, $\sigma$, developed in a rotating solid disc of radius $b$ is given by

$$
\sigma=\frac{(3+\mu)}{8} \delta b^{2} \omega^{2}
$$

where $\delta$ is the density and $\mu$ is the Poisson ratio. The time needed for elimination from the solar system by radiation pressure as a consequence of successive bursting can be estimated by multiplying the time to the first burst by 2.7 (Paddack, 1973).

The time to elimination from the solar system for certain kinds of material by use of Equations 4 and 5 has been calculated and tabulated in Table 1 . The following assumptions were used: heliocentric circular orbits at one astronomical unit, radiation pressure of $4.5 \times 10^{-5}$ dynes $\mathrm{cm}^{-2}$, five gammas for $\mathrm{B}$, (the

Table 1. Elimination time for different materials as a function of particle radius. Magnetic spin damping is included.

Radius

(cm)

$$
10^{-5}
$$

$10^{-4}$

$$
10^{-3}
$$

$10^{-2}$

$10^{-1}$

1
AI

$1.51 \times 10^{1}$

$1.51 \times 10^{2}$

$1.51 \times 10^{3}$

NB

NB

NB
Elimination Time (Years)

$\mathrm{Ni}$

Tektites

$6.25 \times 10^{1}$

$1.49 \times 10^{1}$

6.44

$6.25 \times 10^{2}$

$1.49 \times 10^{2}$

$6.44 \times 10^{1}$

$6.25 \times 10^{3}$

$1.49 \times 10^{3}$

$6.44 \times 10^{2}$

$6.25 \times 10^{4}$

$1.49 \times 10^{4}$

$6.44 \times 10^{3}$

NB

NB

$6.44 \times 10^{4}$

NB

$\mathrm{NB}$

$6.44 \times 10^{5}$ 
interplanetary field intensity (Ness, 1965)), standard resistivity for the metals and $3 \times 10^{-14} \mathrm{ohm}-\mathrm{cm}$ for tektites (Hoyte et al. , 1965). In the same table those particles which reach equilibrium below bursting angular speed are indicated as NB (No Bursting).

\section{Summary}

It has been shown that solar radiation pressure can cause rotational bursting of asymmetric interplanetary dust particles and eliminate them from the solar system. This study predicts a particle lifetime between 10 and $10^{5}$ years, depending critically on the particle radius. It is apparent that due to magnetic damping, this windmill effect does not work for metallic particles whose radii are greater than about $10^{-2} \mathrm{~cm}$. For non-metallic particles, such as tektites, the retarding torque is so small that they will burst in about 6.4 to about $6.4 \mathrm{x}$ $10^{5}$ years and there will be no effective spin stabilization. Comparison with the Poynting-Robertson effect shows that elimination time due to the windmill effect is at least one order of magnitude faster except for those metallic which reach equilibrium $\left(10^{-2} \mathrm{~cm}\right.$ radius or larger). This time ratio is independent of heliocentric distance and particle radius. This might be one of the many reasons " why the spatial density of small solid bodies in interplanetary space is much lower than previously estimated. 


\section{References}

Hoyte, A., F. Senftie, and P. Wirtz, Electrical Resistivity and Viscosity of Tektite Glass, J. Geophys., Res., , 70, 1985-1994, 1965.

Kresák, Ľ. , Structure and Evolution of Meteor Streams, in Physics and Dynamics of Meteors, edited by Ľ. Kresák and P. M. Millman, pp. 391403, D. Reidel Pub. Co., Dordrecht, Holland, 1968.

Ness, Norman F., The Interplanetary Medium, Introduction to Space Science, edited'by Hess, 'Wilmot N., pp. 323-346, New York: Gordon and Breach, 1965.

Paddack, Stephen J., Rotational Bursting of Small Celestial Bodies: Effects of Radiation Pressure, J. Geophys. Res., 74, No. 17, pp. 4379-4381, 1969.

Paddack, Stephen J., Rotational Bursting of Small Celestial Bodies: Effects of Radiation Pressure, Ph. D. Thesis, Catholic University of America, Washington, D. C., 1973.

Radzievskii, V. V., A Mechanism of the Disintegration of Asteroids and Meteorites, Dokl. Akad. Nauk SSSR, 97 (1), pp. 49-52, 1954.

Smythe, W. R., Static and Dynamic Electricity, McGraw-Hill Book Company, New York, 1950.

Timoshenko, S., Strength of Materials, D. Van Nostrand; Princeton, N. J., 1942. 